Commentary

\title{
Linking Klotho, Nrf2, MAP kinases and aging
}

\author{
Priya Balasubramanian and Valter D. Longo \\ Andrus Gerontology Center, University of Southern California, 3715 McClintock Avenue, Los Angeles, CA 90089, \\ USA
}

Commentary on: Hsieh CC, Kuro-o M, Rosenblatt KP, Brobey $R$, and Papaconstantinou J. The ASK1-Signalosome regulates $p 38$ MAPK activity in response to levels of endogenous oxidative stress in the Klotho mouse models of aging. Aging. 2010; 2:597-611

E-mail: vlongo@usc.edu

Oxidative damage of DNA, proteins, lipids and other molecules is one of the oldest mechanisms proposed to explain the phenomenon of aging [1, 2]. Reactive oxygen species (ROS) are generated in the mitchondria as by-products of normal aerobic respiration and in the cytosol by oxidases such as NADPH oxidase. It has been proposed that a decline in mitochondrial function leads to aging because of higher ROS production and increased damage to macromolecules [1]. This is debatable, however, because age-dependent mitochondrial DNA mutations can promote aging without causing increased ROS production [2]. High ROS levels can also regulate signaling via the stress activatedkinases, p38 and JNK. These pathways are often activated in aged tissues and contribute to the inflammation, apoptosis and senescence that is associated with old age. For example, JNK is activated in the liver of old rats and p38 is activated in the liver and brain of old rats as well as in the liver of old mice $[3,4]$.

In the September issue of Aging, $\mathrm{C}-\mathrm{C}$ Hseih et al. report that the p38 kinase is activated in Klotho -/- mice and suppressed by Klotho overexpression [5]. This is dependent on the oxidation and dissociation of Ask-1 bound thioredoxin to generate the active Ask1signalosome which, as previously shown, is an activator of p38 [6]. Klotho overexpressing mice live longer and are characterized by their resistance to oxidative stress [7]. Previous studies by $\mathrm{C}-\mathrm{C}$ Hseih et al. have shown that the ASK1-signalosome-p38 pathway is suppressed in the long-lived and stress-resistant Ames and Snell dwarf mice $[6,8]$. The current study demonstrates that a similar suppression of p38 signaling occurs in Klotho overexpressing cells and provides further evidence that oxidative stress, p38 activity and lifespan are closely linked.
Interestingly, this study also reveals that the stressprotective transcription factor $\mathrm{Nrf} 2$ localizes to the nucleus in Klotho overexpressing cells. Nrf2 is activated by oxidative stress and enhances transcription of genes containing an antioxidant response element (ARE). It is also believed to play an important role in some of the protective effects caused by caloric restriction and by GH/IGF-I deficiencies [9, 10]. Also, thioredoxin which is an inhibitor of Ask1, is a target of Nrf2 [11]. This implies that $\mathrm{Nrf2}$ can regulate the expression of thioredoxin in Klotho mice thereby inhibiting the Ask1-signalosome-p38 pathway. Thus it is possible that the beneficial effects of Klotho are mediated, at least partially, by inactivation of p38 signaling.

The premature aging phenotype of Klotho -/- mice has been attributed to the absence of Klotho mediated suppression of the Insulin/IGF-I pathway [7, 12]. Klotho can activate the transcription factor FOXO, causing up-regulation of the mitochondrial MnSOD [7]. Because many of these cellular phenotypes are also promoted by reduced IGF-I signaling, the new study by $\mathrm{C}-\mathrm{C}$ Hseih et al. not only confirms the major overlap between the pro-aging effects of Klotho -/- and IGF-I but also adds new players that can help identify common downstream anti-aging mechanisms.

\section{REFERENCES}

1. Harman D. Aging: a theory based on free radical and radiation chemistry. J Gerontol. 1956; 11:298-300.

2. Trifunovic A. et al. Somatic mtDNA mutations cause aging phenotypes without affecting reactive oxygen species production. Proc Natl Acad Sci U S A. 2005; 102:17993-17998.

3. Suh $Y$. Age-specific changes in expression, activity, and activation of the c-Jun $\mathrm{NH}(2)$-terminal kinase and p38 mitogenactivated protein kinases by methyl methanesulfonate in rats. Mech Ageing Dev. 2001; 122:1797-1811. 
4. Hsieh CC and J. Papaconstantinou J. The effect of aging on p38 signaling pathway activity in the mouse liver and in response to ROS generated by 3-nitropropionic acid. Mech Ageing Dev, 2002; 123:1423-1435.

5. Hsieh CC et al. The ASK1-Signalosome Regulates p38 MAPK Activity in Response to Levels of Endogenous Oxidative Stress in the Klotho Mouse Models of Aging. Aging. 2010; 2:597-611.

6. Hsieh CC and Papaconstantinou J. Dermal fibroblasts from long-lived Ames dwarf mice maintain their in vivo resistance to mitochondrial generated reactive oxygen species (ROS). Aging 2009; 1:784-802.

7. Yamamoto $\mathrm{M}$ et al. Regulation of oxidative stress by the antiaging hormone klotho. J Biol Chem. 2005; 280:38029-38034.

8. Hsieh CC and Papaconstantinou J. Thioredoxin-ASK1 complex levels regulate ROS-mediated p38 MAPK pathway activity in livers of aged and long-lived Snell dwarf mice. FASEB J. 2006; 20:259-268.

9. Pearson $\mathrm{KJ}$ et al. Nrf2 mediates cancer protection but not prolongevity induced by caloric restriction. Proc Natl Acad Sci U S A. 2008; 105:2325-2330.

10. Sun LY et al. Hepatic response to oxidative injury in longlived Ames dwarf mice. FASEB J. 2010 [Epub ahead of print]

11. Kim YC et al. Hemin-induced activation of the thioredoxin gene by Nrf2. A differential regulation of the antioxidant responsive element by a switch of its binding factors. J Biol Chem. 2001; 276:18399-18406.

12. Kurosu $\mathrm{H}$ et al. Suppression of aging in mice by the hormone Klotho. Science. 2005; 309:1829-1833. 